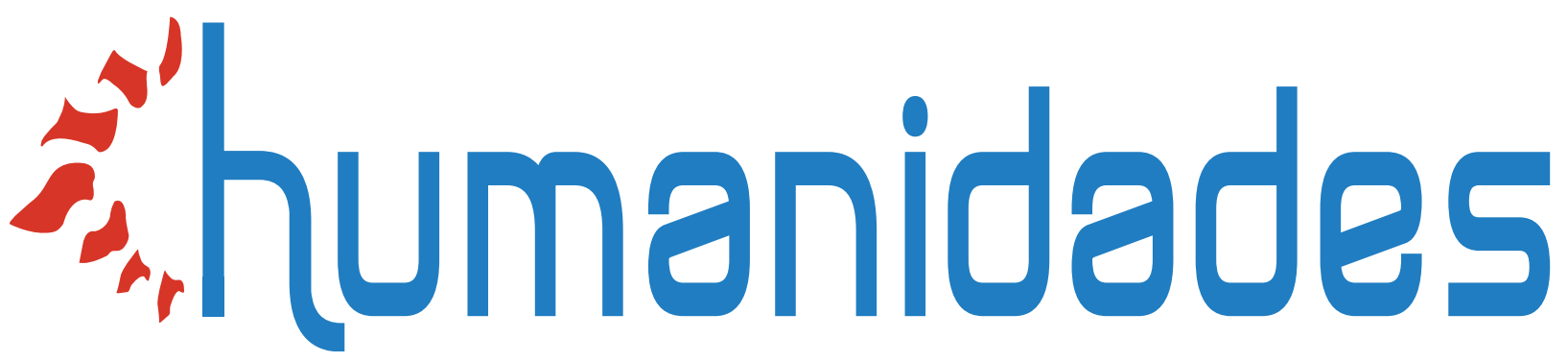

¿Dónde está la presidenta? La construcción de la imagen de Laura Chinchilla en la prensa escrita

\author{
José Andrés Díaz González
}

DOI: https://doi.org/ I0.155 I7/h.vI0il .39762 https://revistas.ucr.ac.cr/index.php/humanidades/index 


\title{
"humanidades
}

Revista Humanidades

ISSN: 2215-3934

humanidades@ucr.ac.cr

Universidad de Costa Rica

Costa Rica

\section{¿Dónde está la presidenta? La construcción de la imagen de Laura Chinchilla en la prensa escrita}

\author{
Díaz González, Dr. José Andrés \\ ¿Dónde está la presidenta? La construcción de la imagen de Laura Chinchilla en la prensa escrita \\ Revista Humanidades, vol. 10, núm. 1, 2020 \\ Universidad de Costa Rica, Costa Rica \\ Disponible en: http://www.redalyc.org/articulo.oa?id=498060395012 \\ DOI: https://doi.org/10.15517/h.v10i1.39762
}

Todos los derechos reservados. Universidad de Costa Rica. Esta revista se encuentra licenciada con Creative Commons. Reconocimiento-NoComercial-SinObraDerivada 3.0 Costa Rica. Correo electrónico: humanidades@ucr.ac.cr/ Sitio web: http: //revistas.ucr.ac.cr/index.php/humanidades

Esta obra está bajo una Licencia Creative Commons Atribución-NoComercial-SinDerivar 3.0 Internacional. 


\section{¿Dónde está la presidenta? La construcción de la imagen de Laura Chinchilla en la prensa escrita}

Where is the President? The Construction of the Image of Laura Chinchilla in the Press

Dr. José Andrés Diaz González

Instituto de Estudios Sociales en Población, Universidad

Nacional de Costa Rica, Costa Rica

DOI: https://doi.org/10.15517/h.v10i1.39762

Redalyc: http://www.redalyc.org/articulo.oa?

jose.diaz.gonzalez@una.ac.cr

(iD http://orcid.org/0000-0002-6063-086X id $=498060395012$

Recepción: 25 Octubre 2019

Aprobación: 22 Noviembre 2019

\section{Resumen:}

Este artículo analiza la imagen que se construye de Laura Chinchilla Miranda, durante su primer año de mandato, por la prensa escrita. Para ello, se recolectan noticias publicadas entre el 8 de mayo del 2010 y el 7 de mayo del 2011, sobre cuatro conflictos sociales en los que debe intervenir la Presidencia de la República. Se concluye que las noticias tienden a presentar a Chinchilla Miranda como una figura ausente o pasiva, e incluso en muchas ocasiones retratan o relatan sus acciones a partir de lo expresado por los hombres que integraban su gabinete.

Palabras Clave: Prensa, Conflicto Social, Política, Costa Rica.

\section{Abstract:}

This article analyzes the image of Laura Chinchilla Miranda during her first year in office, in the written press. To do this we collect the news published between May 8, 2010, and May 7, 2011, about four social conflicts in which the Presidency of the Republic must intervene. In conclusion, the news presents to Chinchilla Miranda as an absent or passive figure, and even frequently, they portray their actions from what was expressed by the men of her cabinet.

Keywords: Press, Social Conflict, Politics, Costa Rica.

El Análisis Crítico del Discurso (ACD), como lo ha indicado Teun van Dijk en distintos momentos (1999, 2000, 2003a y 2003b), tiene el propósito de identificar y denunciar de qué manera se utilizan los distintos discursos para la reproducción de estructuras de poder y dominación en perjuicio de distintos grupos sociales, los cuales quedan relegados u oprimidos por otros sujetos por medio de dichas estructuras. Así, podría parecer poco acertado recurrir al ACD para tratar de estudiar el discurso de los medios de comunicación con respecto a la persona que se encuentra en la presidencia de la república, esto debido a que puede pensarse que esta persona, dado su cargo político, se encuentra de lado de los grupos dominantes que controlan las estructuras de poder.

Sin embargo, al utilizar el ACD para estudiar los discursos de la prensa escrita con respecto a Laura Chinchilla Miranda, quien ejerció la presidencia de la república de Costa Rica durante el período 2010-2014, se puede visualizar la forma en la que en estos discursos se siguen reproduciendo tensiones sociales (ideológicas, de género, etc.) que afectan la forma en que se considera la imagen de la presidenta y su participación en el espacio público. Asimismo, desde el ACD se puede examinar cómo esta construcción discursiva de parte de los medios de comunicación responde a una estrategia desarrollada por estos para impulsar, ante la opinión pública, su propia perspectiva con respecto a la forma en que debe estructurarse el poder en el país; en otras palabras, potenciar su propia visión política. 
Desde el ACD, el análisis de un solo párrafo puede extenderse en varias decenas o, incluso, centenas de páginas; de esta forma, el análisis de una cantidad de centenares de noticias, como las recolectadas para la presente investigación, en algunas decenas de páginas de forma profunda y completa es una tarea que raya en lo imposible. Por lo tanto, el análisis de estos textos que se presentan no busca ni pretende ser exhaustivo, sino que su intención es identificar las líneas o tendencias generales del discurso acerca de Laura Chinchilla Miranda presente en las noticias recolectadas.

Para realizar el análisis de las noticias se parte de la propuesta de van Dijk (2002) presente en su texto La multidisciplinariedad del análisis crítico del discurso: un alegato en favor de la diversidad, en el cual expone algunas directrices que sigue cuando realiza el ACD. Esencialmente van Dijk indica la necesidad de realizar el ACD de un texto, a partir de la consideración de las estructuras contextuales, cognitivas y discursivas en el que se circunscribe este. Esta investigación se concentra en el análisis de las estructuras contextuales y discursivas; en cuanto las estructuras cognitivas, algunos aspectos sobre la ideología y elementos cognitivos que están presentes en la población costarricense para la interpretación del discurso estudiado, se encuentran presentes en el apartado de conclusiones; no obstante, solamente se dispone de algunas consideraciones básicas, quedando pendiente un análisis más profundo de las estructuras cognitivas que se relacionan con el discurso de la prensa escrita con relación a la imagen de Laura Chinchilla Miranda.

Por lo anterior, se propone revisar de qué forma el discurso de la prensa escrita ha presentado la imagen de Laura Chinchilla en el marco de cuatro conflictos sociales, los cuales debe enfrentar durante su primer año de gestión (del 8 de mayo del 2010 al 7 de mayo del 2011). El propósito es determinar qué imagen de la presidenta buscan transmitir estos periódicos a la población.

\section{Aspectos Metodológicos}

Para la elaboración del análisis se procedió, en primera instancia, con la recolección de todas las noticias publicadas por los periódicos seleccionados entre el 8 de mayo del 2010 y el 7 de mayo del 2011, relacionadas, directamente, con: el proyecto minero Crucitas, el proceso de concesión de los puertos de JAPDEVA, el intento de llevar a cabo un referéndum sobre la legalización de las uniones civiles de personas del mismo sexo y la negociación del FEES (Fondo Especial para la Educación Superior). En total se recolectaron 706 noticias en las cuales los medios de comunicación abordaban dichos temas. El motivo por el que fueron seleccionados estos casos se debe a que todos fueron seguidos por La Nación, Diario Extra, El País y el Semanario Universidad (ver Tabla 1); asimismo, en estos conflictos -por su naturaleza- se esperaba observar una participación activa de la presidenta, que permitiera identificar cómo los medios retratan su relación con respecto a otros actores políticos, así como con los distintos movimientos y grupos sociales del país. 
TABLA 1

Caracterización de los medios de comunicación revisados

\begin{tabular}{|l|l|}
\hline La Nación & $\begin{array}{l}\text { Periódico que pertenece al Grupo Nación S.A. } \\
\text { Fundado en octubre de 1946, se publica de } \\
\text { forma diaria. Tiende a identificarse con los } \\
\text { intereses de los grupos de la clase alta en los } \\
\text { planos tanto económico como político de Costa } \\
\text { Rica. }\end{array}$ \\
\hline Diario Extra & $\begin{array}{l}\text { Es un tabloide que se publica de lunes a } \\
\text { sábado. Fundado en septiembre de 1978, } \\
\text { posee un enfoque amarillista en las notas que } \\
\text { provee. }\end{array}$ \\
\hline El País & $\begin{array}{l}\text { Uno de los primeros periódicos digitales de } \\
\text { Costa Rica. Inclinado a detentar un enfoque } \\
\text { favorable hacia los movimientos y las críticas } \\
\text { sociales en general. }\end{array}$ \\
\hline Semanario & $\begin{array}{l}\text { Fundado en 1970; este medio pertenece a la } \\
\text { Universidad de costa Rica (UCR), y tiende a } \\
\text { tener un enfoque crítico y concentrado en los } \\
\text { problemas políticos, sociales y económicos a } \\
\text { nivel nacional. }\end{array}$ \\
\hline
\end{tabular}

Fuente: Elaboración propia.

Posteriormente, se procedió a identificar las noticias en las que dentro de su contenido se mencionaba de forma directa o indirecta- a Laura Chinchilla, siendo estas un total de 161. Por último, se definieron las unidades de análisis:

1. Los temas centrales ubicados en los párrafos o segmentos del texto de las noticias en las que se mencionaba a Chinchilla Miranda.

2. Las palabras presentes en el texto que permitieran identificar la presencia o que hiciera referencia a la figura de la presidenta (ver Tabla 2).

TABLA 2

Universo, unidades de observación y unidades de análisis identificadas para el análisis de contenido

\begin{tabular}{|l|l|}
\hline Universo & $\begin{array}{l}\text { Todas las noticias publicadas por La Nación, } \\
\text { Diario Extra, El País y el Semanario } \\
\text { Universidad, en sus versiones digitales, sobre } \\
\text { los conflictos sociales seleccionadas, entre el } 8 \\
\text { de mayo del 2010 y el 7 de mayo del 2011. }\end{array}$ \\
\hline $\begin{array}{l}\text { Unidades de } \\
\text { Observación }\end{array}$ & $\begin{array}{l}\text { Las noticias publicadas por los medios } \\
\text { estudiados sobre los conflictos sociales } \\
\text { seleccionados, en los que se haga mención de } \\
\text { Laura Chinchilla Miranda. }\end{array}$ \\
\hline Unidades de & $\begin{array}{l}\text { 1) Temas contenidos en los fragmentos de texto } \\
\text { de las noticias en los que se menciona a } \\
\text { Chinchilla Miranda 2) Palabras vinculadas o } \\
\text { relacionadas con la presencia e imagen de } \\
\text { Chinchilla Miranda. }\end{array}$ \\
\hline
\end{tabular}

Fuente: Elaboración propia.

Debe indicarse que la información recopilada corresponde únicamente a noticias y que no incluye a los grupos editoriales ni los artículos de opinión publicados durante ese período por dichos medios de comunicación relacionados con los conflictos seleccionados. Esto debido a que lo que se busca es observar cómo cada medio noticioso presenta la información que hace referencia a este caso, por lo que utilizar 
los artículos de opinión publicados en los medios (periódicos) provocaría que se analizara el contenido de posiciones que no necesariamente corresponden a la visión del medio.

Para analizar la información se recurrió al análisis de contenido, el cual es “[...] una técnica de investigación destinada a formular, a partir de ciertos datos, inferencias reproducibles y válidas que puedan aplicarse a su contexto" (Krippendorff, 1997, p. 28). Si bien el análisis de contenido es una técnica cualitativa, es necesario señalar que le subyace una aproximación cuantitativa, a partir de la cual se procura lograr la descripción objetiva y sistemática del contenido manifiesto de los textos ${ }^{1}$. Es necesario aclarar que la característica de objetividad que se le atribuye al análisis de contenido no debe entenderse como una renuncia a las apreciaciones subjetivas, ni mucho menos como una condena hacia estas; sino que tiene que ver con la necesidad que poseen todas las personas que realizan una investigación de contar con una serie de procedimientos razonados y ejecutados a partir de los cuales son sustentados sus hallazgos (Abarca, 2012, pp. 194-195).

Es por eso que para la construcción del análisis de las noticias se parte de una base cuantitativa, es decir, del conteo del número de apariciones de Chinchilla Miranda en las noticias, de los diferentes temas con los que se relaciona su aparición, de la cantidad de opiniones de la presidenta expresas en los textos, etc. No obstante, en ningún momento se pretende realizar un análisis cuantitativo o estadístico de dichos datos, sino que se procuran sirva de base para desarrollar un análisis cualitativo que permita identificar la imagen de Laura Chinchilla que los medios de comunicación buscan proyectar hacia la opinión pública. Asimismo, es necesario indicar que el análisis de contenido facilita identificar cómo los medios presentan la información sobre un mismo acontecimiento, ampliando la comprensión de la forma en la que a través de estos textos los medios proporcionan a sus lectores material para la construcción de su sentido de "contexto"; en otras palabras, este tipo de análisis permite observar de qué manera la organización de unidades básicas del texto, como las palabras, la elección entre voz activa y voz pasiva y la yuxtaposición de discursos encontrados, pueden servir como base para la construcción de discursos de los que una determinada estructuración del poder en la sociedad sea presentado como algo "natural" para las personas lectoras (West, Lazar y Kramarae, 200, p. 190).

\section{Estructura conteXtual: El CONTEXTO EN EL QUE SE CONSTRUYE LA FigurA De LA PRESIDENTA.}

Para comprender el discurso de los medios informativos sobre Laura Chinchilla es necesario referirnos al marco contextual en el que se enmarca este discurso ya que, como indica van Dijk (2000, pp. 32-33 y 2003a, p. 160) el discurso se produce, comprende y analiza en relación con las características del contexto; el cual puede definirse como la estructura de aquellas propiedades de la situación social que son sistemáticamente relevantes para el discurso. Asimismo, este autor hace una distinción con respecto a los contextos globales y los locales en los cuales se produce el discurso; el primero, hace referencia a aquel contexto más social o abstracto, mientras que el segundo, hace alusión a aquellos aspectos más cercanos con los cuales interacciona el discurso (van Dijk, 2000, p. 37 y 2003a, p. 161).

Así, para el caso en el que se analiza el contexto global se contienen los elementos relacionados con los procesos sociopolíticos y con los acontecimientos que han afectado o promovido la participación de las mujeres en el espacio político, más precisamente, aquellos que de una u otra forma se vinculan con la elección de la primera mujer en la presidencia de la república. En cuanto el contexto local, este hace referencia a los acontecimientos, actores y acciones relacionadas con los cuatro casos de conflictos sociales que se han mencionado previamente con el propósito de estudiar el contenido de las noticias publicadas por los medios con respecto a estos.

En cuanto el contexto global, en primera instancia, es necesario enmarcar la designación de Chinchilla Miranda como presidenta de la República de Costa Rica en el contexto de una "segunda ola" de designación de mujeres en dicho cargo en América Latina. La "primera ola" de designación de mujeres como presidentas 
ocurre en las décadas finales del siglo XX, con la llegada a dichos puestos de Violeta Barrios (Nicaragua) y Mireya Moscoso (Panamá); y se caracteriza porque su llegada al poder se debió a sus vínculos personales y familiares o a momentos de inestabilidad política (Fernández y Oliva, 2012, pp. 119-120).

Por su parte, la "segunda ola" de designaciones de mujeres como presidentas de la república ocurre dentro de un marco de fortalecimiento tanto de los derechos políticos de las mujeres, como de profundización o fortalecimiento de la democracia en los países en donde estas han sido electas (Chile, Argentina, Brasil y Costa Rica). Asimismo, este proceso de profundización de los derechos políticos de las mujeres no es el único aspecto que ha facilitado que hayan llegado a ganar las elecciones presidenciales; sino que es necesario destacar que son mujeres que cuentan con su propia trayectoria política, además de cierta independencia ante los grupos de poder dominantes (Fernández y Oliva, 2012, pp. 120-122). Así, Chinchilla Miranda viene a engrosar un grupo en el cual se encuentran otras mujeres latinoamericanas que llegan a ocupar la silla presidencial: Michelle Bachelet (2006-2010), Cristina Fernández (2007-2015) y Dilma Rousseff (2011-2015). Por lo tanto, su llegada a la presidencia se debe a que cuentan con suficientes méritos políticos para optar por dicho cargo; lo cual no implica que no contaran con el apoyo de algún grupo político o no se identificaran con alguna tendencia política que les sirviera como apoyo para llegar a la Presidencia (Navia, 2007 y Morales, 2007).

En el plano costarricense, Chinchilla es la primera mujer en la historia del país en ser designada para ocupar la silla presidencial. Si bien dicho acontecimiento puede observarse como todo un logro, al mismo tiempo, es un desafío. En un país en el que las mujeres obtuvieron el derecho a elegir y ser electas justo a mediados del siglo pasado y que además se enorgullece por su "centenaria tradición democrática”, el camino de las mujeres por lograr una mayor participación en el espacio político, bajo las mismas condiciones que los hombres, no ha sido una tarea fácil en estos últimos 60 años, y aún está lejos de ser una tarea concluida. Si bien la elección de Laura Chinchilla como presidenta de la república puede dar la sensación de que se ha alcanzado una relativa igualdad en el plano político, una rápida revisión de las estadísticas de los puestos políticos de elección popular que han sido ocupados por mujeres durante el siglo XX e inicios del XXI muestra una realidad distinta.

En cuanto el contexto local, este se relaciona directamente con los actores los acontecimientos, y las acciones ocurridas en los cuatros conflictos sociales seleccionados: el desarrollo de un proyecto de minería a cielo abierto en la comunidad de Crucitas, la posible realización de un referéndum sobre la legalización de las uniones civiles de personas del mismo sexo, el proceso de privatización de los puertos del Caribe, administrados por JAPDEVA, y la negociación del FEES.

El primer conflicto, se relaciona con el desarrollo de un proyecto de minería de oro a cielo abierto en la comunidad de Crucitas, ubicada al norte de Costa Rica. Si bien el inicio del descontento social en relación con este proyecto puede rastrearse hasta el año 2001 con el otorgamiento de la concesión minera, el punto que marca el inicio de la mayor conflictividad ocurre en el 2009, cuando Óscar Arias Sánchez, entonces presidente de Costa Rica, firma un decreto declarando de interés nacional el desarrollo del proyecto minero. Al llegar Laura Chinchilla a la presidencia de Costa Rica, debe hacer frente a múltiples manifestaciones sociales (huelgas de hambre, caminatas, protestas, etc.) con el propósito de solicitar la anulación de la declaratoria realizada por Arias Sánchez y detener el desarrollo del proyecto. El 24 de noviembre del 2010, cuando el Tribunal Contencioso Administrativo anula la concesión minera por incumplir con una serie de requisitos y permisos, se marca la finalización del punto más álgido de la movilización social surgida en torno a este conflicto.

Por su parte, el segundo conflicto, nace con el intento de llevar a cabo un referéndum -en el 2010- para que fuera la población costarricense la que decidiera si se aprobaba o no el proyecto de ley No.16.390, dicho proyecto de ley pretendía la legalización de las uniones civiles de personas del mismo sexo. Al respecto, desde el 2009 la organización Observatorio Ciudadano por la Vida y la Familia, grupo afín a la jerarquía de la Iglesia Católica costarricense, promovía la realización del referéndum como una estrategia para evitar que dicho proyecto de ley fuera aprobado en la Asamblea Legislativa. Lo anterior, llevó a grupos en defensa de 
los derechos humanos, estudiantes, universidades públicas y a la Defensoría de los Habitantes a promover acciones ante el Tribunal Supremo de Elecciones (TSE), con el objetivo de frenar dicha consulta popular. En cuanto Chinchilla, durante la campaña política por la presidencia de la república, se manifestó abiertamente en contra de legalizar las uniones entre personas del mismo sexo y, al llegar a la silla presidencial, indicó que el tema no era prioritario para su administración por lo que no tomaría partido sobre la realización del referéndum y acataría la decisión adoptada por este. Al final, el referéndum no es llevado a cabo debido a que la Sala Constitucional, en junio del 2010, declara con lugar un recurso de amparo presentado contra dicha consulta, argumentando que la legislación sobre derechos humanos no podía ser sometida a dicho mecanismo político.

El tercer conflicto seleccionado ocurre en relación con el intento de privatizar los puertos administrados por la Junta de Desarrollo de los Puertos de la Vertiente Atlántica (JAPDEVA). El conflicto tiene sus inicios en la Administración Arias Sánchez (2006-2010), cuando Arias anuncia la intención de dar los puertos en concesión a una empresa holandesa; como respuesta, el Sindicato de Trabajadores de JAPDEVA (SINTRAJAP) le envía al Gobierno una propuesta de modernización de los puertos, la cual no requería su privatización. A partir de ahí empieza una serie de conflictos entre el Gobierno y el SINTRAJAP; situación que empeora a inicios del 2010, cuando un grupo de trabajadores, apoyados por el Gobierno, deponen de manera irregular a la junta directiva del sindicato y en su lugar colocan una junta directiva afín al proyecto de privatización. Esto provoca que tanto la junta directiva depuesta, grupos de trabajadores de JAPDEVA, partidos políticos y otros sindicatos costarricenses, lleven a cabo una serie de protestas y acciones, tanto con el objetivo de reinstaurar a la junta directiva anterior de SINTRJAP, como para solicitarle a la administración Chinchilla Miranda que detenga el proceso de privatización. En agosto del 2010 la Sala Constitucional obliga la reinstalación de la junta directiva anterior y, en enero del 2011 dicha junta es reelecta; lo cual marca la detención -temporal- del proyecto de concesión portuaria.

El último conflicto, sobre el que se recolectan noticias, se relaciona con el proceso de negociación del Fondo Especial de Educación Superior (FEES), el cual es el mecanismo de financiamiento de las universidades públicas en Costa Rica. Las negociaciones inician en junio del 2010, entre los rectores y rectoras de las universidades públicas y los ministros de Educación, Hacienda y la ministra de Ciencia y Tecnología. No obstante, el poco avance en estos procesos de negociación lleva a la comunidad universitaria a realizar una serie de acciones de protesta con el propósito de solicitar a Chinchilla que intervenga directamente en el proceso de negociación. En agosto del 2010, las universidades públicas y el Gobierno llegan a un acuerdo sobre el monto del presupuesto con las que estas serán financiadas durante los siguientes cinco años.

\section{Estructura discursiva: LA IMAgen de LAURA Chinchilla EN EL Discurso De LA PRENSA ESCRITA}

Siguiendo a van Dijk, se entiende discurso en el amplio sentido de "acontecimiento comunicativo", incluyendo "[...] la interacción conversacional, los textos escritos y también los gestos asociados, el diseño de portada, la disposición tipográfica, las imágenes y cualquier otra dimensión o significación "semiótica" $o$ multimedia" (van Dijk, 2003a, p. 146). Lo anterior, presenta la disyuntiva de qué elementos y niveles serán seleccionados para llevar a cabo el análisis del discurso. Como se indicó al inicio, lo que interesa es realizar un análisis general de todas las noticias publicadas con el propósito de observar cuál es la imagen de Chinchilla Miranda que buscan transmitir, por lo tanto, nos concentramos en el estudio de las macroestructuras semánticas; lo cual permite -por medio del estudio de elementos tales como titulares, fragmentos, oraciones, resúmenes, temas, entre otros- identificar los significados globales que el texto busca transmitir, así como su relación con la estructura contextual y cognitiva que acompaña a este (van Dijk, 2003a, pp. 152-153).

Como ya se indicó, para la realización del presente análisis se procedió primero a recolectar el número total de noticias publicadas por La Nación, Diario Extra, El País y el Semanario Universidad, con respecto a 
los cuatro conflictos sociales seleccionados (ver Cuadro 1). No obstante, no en todas las noticias publicadas aparece o se hace referencia a Laura Chinchilla, por lo cual, para poder determinar qué tanto es destacada ella en el contenido publicado por los medios de comunicación, se procede primero a identificar en cuantas noticias es mencionada. En total, Chinchilla Miranda es mencionada solamente en 161 de las 706 noticias publicadas por los medios sobre estos temas (ver Cuadro 2), es decir, aparece únicamente en menos de la cuarta parte de las noticias (22,8\%). Este dato es revelador, ya que muestra que las opiniones o las acciones de la presidenta no son elementos centrales en la información proporcionada por los periódicos estudiados.

CUADRO 1

Número de noticias publicadas sobre los casos seleccionados por medio de comunicación estudiado

\begin{tabular}{llllll} 
& Crucitas & JAPDEVA & FEES & $\begin{array}{l}\text { Unión civil de } \\
\text { personas del } \\
\text { mismo sexo }\end{array}$ & Total \\
\hline La Nación & 63 & 47 & 30 & 24 & 164 \\
Diario Extra & 43 & 64 & 35 & 22 & 164 \\
Semanario & 18 & 12 & 43 & 4 & 77 \\
Universidad & & & & & 301 \\
El País & 212 & 62 & 7 & 20 & 706 \\
Total & 336 & 185 & 115 & 70 & \\
\hline
\end{tabular}

Fuente: Elaboración propia, a partir de las noticias encontradas en La Nación, Diario

Extra, El País y el Semanario Universidad, del 8 de mayo del 2010 al 7 de mayo del 2011.

CUADRO 2

Cantidad de noticias en las que se menciona a Laura Chinchilla, según medio analizado

\begin{tabular}{|c|c|c|c|c|c|}
\hline & \multicolumn{4}{|l|}{ Medio } & \multirow[b]{2}{*}{ Total } \\
\hline Conflicto & La Nación & $\begin{array}{l}\text { Diario } \\
\text { Extra }\end{array}$ & El País & Semanario & \\
\hline JAPDEVA & 10 & 8 & 14 & 4 & 36 \\
\hline FEES & 7 & 13 & 9 & 2 & 31 \\
\hline $\begin{array}{l}\text { Proyecto minero } \\
\text { Crucitas }\end{array}$ & 15 & 14 & 54 & 5 & 88 \\
\hline $\begin{array}{l}\text { Referéndum sobre } \\
\text { unión civil de personas } \\
\text { del mismo sexo }\end{array}$ & 1 & 0 & 4 & 1 & 6 \\
\hline Total & 33 & 35 & 81 & 12 & 161 \\
\hline
\end{tabular}

Fuente: Elaboración propia, a partir de las noticias encontradas en La Nación, Diario

Extra, El País y el Semanario Universidad, del 8 de mayo del 2010 al 7 de mayo del 2011.

El medio que da un mayor seguimiento, de forma proporcional, a las acciones de la presidenta es periódico El Pais, en total el $26,9 \%$ de sus noticias (81 noticias) mencionan a Laura Chinchilla. Asimismo, en las noticias en las que más se menciona de forma proporcional a Chinchilla son aquellas que se relacionan con el proceso de negociación del FEES, ya que ella aparece en el 26,9\% (31 noticias) de las noticias vinculadas a este tema; seguido muy de cerca por las noticias relacionadas con el proyecto minero Crucitas, donde se le menciona en el $26,1 \%$ de las publicaciones ( 88 noticias). Donde tiene una menor aparición es en las noticias publicadas con respecto a la posibilidad de realizar un referéndum sobre la legalización de la unión de personas del mismo sexo, ya que solo se la menciona en el $8,5 \%$ de las noticias. 
TABLA 3

Titulares en los que se menciona a Laura Chinchilla en los medios estudiados

\begin{tabular}{ll} 
Medio & Titulares \\
\hline "Sindicato de JAPDEVA defraudó a Chinchilla". "Chinchilla llama a \\
apurar negociación por presupuesto universitario". "Diputados exigen \\
a Chinchilla resolver diferencias con el FEES". "Oposición exige a \\
Chinchilla resolver conflicto con el FEES". "Chinchilla estudia decreto \\
sobre minería en Crucitas". "Chinchilla toma más dias para definir \\
Sobre Crucitas". \\
"Chinchilla pide respaldo para concesión portuaria". \\
"Dirigencia legitima de SINTRAJAP solicita reunión con presidenta \\
Chinchilla". "Chinchilla trata de remover obstáculos para modernizar \\
puertos". "José Merino: En Costa Rica, Laura Chinchilla está \\
gobernando para los ricos". "Mineria, seguridad y política social, \\
primeros decretos de Chinchilla". "Crucitas queda por fuera del decreto \\
minero de la nueva presidenta". "PARLACEN demanda a presidenta de \\
Costa Rica frenar mineria "Las Crucitas". "Chinchilla continua politica \\
ambiental de los Arias en el SENARA". "PAC pide a presidenta anular \\
Concesión minera en Crucitas". "Chinchilla no dará más permisos para \\
mineria a cielo abierto pero no habla de Crucitas". "Solicitarán a Laura. \\
Chinchilla prohibir el cianuro en actividades mineras". "Laura \\
Chinchilla anunció que Gobierno no anulará el proyecto Crucitas". \\
"Dudas sobre transparencia de Chinchilla en moratoria minera se \\
Confirman". "Presidenta de Costa Rica estudiará fallo judicial que \\
anuló concesión minera". "Caminantes esperaran respuesta de \\
Chinchilla esta tarde". "Minería a cielo abierto de Crucitas en manos de \\
Laura Chinchilla". "Viceministro y asesor de Laura Chinchilla ha sido \\
un aliado de Crucitas". "Inician ayuno indefinido para "apoyar" a Laura \\
Chinchilla en derogación del decreto Crucitas". "PAC pide a Presidente \\
Chinchilla dialogar con ayunantes". "Solicitud a la presidente Laura \\
Chinchilla para eliminar decreto Crucitas". "Legisladores urgen a \\
presidente a Chinchilla a publicar reformas Código Minero". "Laura \\
Chinchilla dedica sus primeros meses de gobierno a "apagar \\
incendios".".
\end{tabular}

Fuente: Elaboración propia, a partir de las noticias encontradas en La Nación, Diario

Extra, El Pais y el Semanario Universidad, del 8 de mayo del 2010 al 7 de mayo del 2011.

Un aspecto aún más revelador surge al revisar los titulares de las noticias en las que es mencionada Chinchilla Miranda. Como mencionan Leñero y Marín (1986. p. 60) los titulares son "[...] "el grito", la llamada de atención con la que los medios anuncian la noticia [el resaltado es original]"; así, estos buscan centrar la atención de la persona lectora en un aspecto o tema particular de la información que el medio busca transmitir, buscando afianzar entonces esa idea o percepción en la imagen que se construye a partir de la lectura del artículo. Así, del total de noticias publicadas en las que se hace alguna mención a Laura Chinchilla, esta aparece mencionada en 27 titulares; es decir, solo en el 16,7\% de las noticias publicadas en que aparece Chinchilla Miranda hubo una intención de los medios de llamar la atención de la persona lectora y enfocarla hacia la imagen de la presidenta. Se observa entonces que El País es el medio en el cual se le da una mayor mención de Chinchilla Miranda en sus titulares, mientras que en el Semanario Universidad no es mencionada en ningún titular (ver Tabla 3).

Es necesario señalar que la selección de la forma en la que se expresa o comunica un mensaje, especialmente en lo concerniente a los medios de comunicación, no puede ser considerada simplemente como una decisión estilística, sino que la forma en que se construye (en este caso redacta) el mensaje tiene el propósito en el discurso informativo de inducir en el público una forma determinada de interpretar dicha información (Fernández, 2005, pp. 220-221 y van Dijk, 2003a, p. 159). Considerando esto, un vistazo a la Tabla 3 permite apreciar que la mención a Laura Chinchilla en los titulares puede clasificarse según su aparición, ya sea en forma activa o pasiva, lo cual puede suponerse como un intento de los periódicos de inducir en las personas 
lectoras una determinada interpretación de la (re)acción de Chinchilla Miranda ante los acontecimientos que informan. En las apariciones como figura activa Laura Chinchilla es el sujeto de la oración, en otras palabras, los medios indican que ella realiza algún acto directamente vinculado con los temas que informan en el contenido de las noticias. Por otra parte, su aparición como figura pasiva indica que ella es el objeto de una acción o solicitud realizada por otro actor, en otras palabras, se trata de resaltar como punto relevante en el contenido informativo que se le solicita a ella a intervenir o a actuar de una manera específica con respecto a los conflictos sociales informados.

CUADRO 3

Clasificación de las menciones a Laura Chinchilla en los titulares de las noticias analizadas

\begin{tabular}{|c|c|c|c|c|c|c|c|}
\hline \multirow{2}{*}{ Conflicto } & \multicolumn{2}{|c|}{ La Nación } & \multicolumn{2}{|c|}{ Diario Extra } & \multicolumn{2}{|l|}{ El País } & \multirow{2}{*}{-Total } \\
\hline & Activo & Pasivo & Activo & Pasivo & Activo & Pasivo & \\
\hline JAPDEVA & 0 & 1 & 1 & 0 & 2 & 1 & 5 \\
\hline FEES & 1 & 2 & 0 & 0 & 0 & 0 & 3 \\
\hline $\begin{array}{l}\text { Proyecto minero } \\
\text { Crucitas }\end{array}$ & 2 & 0 & 0 & 0 & 5 & 11 & 18 \\
\hline $\begin{array}{l}\text { Referéndum sobre } \\
\text { unión civil de } \\
\text { personas del } \\
\text { mismo sexo }\end{array}$ & 0 & 0 & 0 & 0 & 1 & 0 & 1 \\
\hline Total & 3 & 3 & 1 & 0 & 8 & 12 & 27 \\
\hline
\end{tabular}

Fuente: Elaboración propia, a partir de las noticias encontradas en La Nación, Diario

Extra, El Pais y el Semanario Universidad, del 8 de mayo del 2010 al 7 de mayo del 2011.

En el Cuadro 3 se muestra la clasificación de los titulares en los que se menciona a Chinchilla Miranda tanto por tema como por medio, y su clasificación con relación a si ella se presenta como sujeto pasivo o activo. El medio en el que la presidenta es mencionada en mayor medida en sus titulares es en El País, donde aparece en 20 titulares, en la mayoría de los casos (12 titulares) lo hace como sujeto pasivo. El siguiente medio con la mayor cantidad de menciones a Laura Chinchilla es La Nación, en este caso, publica la misma cantidad de titulares donde esta aparece como sujeto activo y pasivo. Por último, Diario Extra publica un único titular en el que ella aparece como sujeto activo.

De forma general, se puede apreciar que hay cierta tendencia de los titulares de los medios de comunicación de presentar a Chinchilla Miranda como un sujeto pasivo. En estos casos, sujetos como partidos políticos, grupos ambientalistas, sindicatos, etc., le solicitan a la presidenta tomar distintas acciones, incluyendo el diálogo con ellos con respecto a los conflictos sociales que se informan en los medios. Esta tendencia puede interpretarse como un intento de los medios de mostrar a Chinchilla Miranda como una figura que no actúa por iniciativa propia con respecto a estos problemas, ya que otros actores deben buscarla y "demandarle" que se pronuncie, actúe o dialogue con ellos acerca de estos temas.

Por lo tanto, se puede concluir que la visibilización de Laura Chinchilla en las noticias publicadas por los medios seleccionados es bastante baja. Si bien es cierto que, dada la naturaleza de los conflictos seleccionados, la presidenta de la república no necesariamente era la agente con mayor capacidad de decisión para solucionar estos problemas, sino que existían otras personas o instituciones que tenían dicha función; es necesario acotar que los medios no realizan necesariamente esa distinción al informar sobre los conflictos. Asimismo, las noticias informan cómo distintos grupos buscan a Chinchilla Miranda para obtener una respuesta a sus demandas respectivas, con lo cual se puede inducir el mensaje de que ella tenía una mayor capacidad de acción ante el conflicto del que realmente poseía.

Un aspecto particularmente llamativo es que los periódicos tienden a presentar a actores masculinos como los interlocutores principales de la postura del Gobierno. El caso más claro ocurre en las noticias publicadas 
en relación al FEES, donde se destacan a los ministros Leonardo Garnier y Fernando Herrero. Si bien es cierto que ambos ministros conformaban la llamada Comisión de Enlace que tenía como objetivo negociar con las universidades públicas el monto del fondo de educación, en esta comisión también se encontraba la ministra de Ciencia y Tecnología, Clotilde Fonseca, la cual es dejada de lado en las publicaciones de los medios; es más, cuando en las noticias se hace la referencia a los "ministros" que participan en dicho proceso es para hacer referencia a Garnier y Herrero, dejando completamente de lado la figura de Fonseca (Díaz, 2012b).

Una situación similar a la anterior ocurre en las noticias publicadas con relación a la posibilidad de realizar un referendo sobre la legalización de la unión civil de personas del mismo sexo. En este caso, Laura Chinchilla es excluida casi completamente de la escena, ya que solo un medio -El Pais- la menciona en sus noticias de forma relevante, y el Semanario Universidad hace un par de menciones puntuales acerca de ella. En contraste, Luis Antonio Sobrado, presidente del Tribunal Supremo de Elecciones (TSE), es el actor proveniente de la institucionalidad pública más mencionado. La falta de visibilización de Chinchilla Miranda sobre este tema es particularmente llamativa debido a que, durante la campaña electoral, manifestó abiertamente su posición en defensa de la familia "tradicional” y en contra de la legalización de las uniones entre personas del mismo sexo; asimismo, ya en el ejercicio de la presidencia de la República, Laura Chinchilla negó la participación de Costa Rica como signataria de la declaración de la Organización de las Naciones Unidas (ONU) que buscaba la despenalización de la homosexualidad, debido a que dicho tema no fue tocado en las negociaciones realizadas por el país en su campaña para presidir el Consejo de Derechos Humanos de la ONU (Díaz, 2012a, p. 313).

En ambos casos mencionados, la estructura discursiva puede enlazarse con lo indicado previamente sobre la estructura contextual, ya que a pesar de que en las últimas décadas se puede observar que de forma cuantitativa se ha profundizado la participación de las mujeres en el campo político costarricense, todavía se está lejos de alcanzar una igualdad real en este campo; lo cual se ve reflejado en la predominancia de los hombres como actores relevantes en el espacio político sobre las mujeres, en el contenido de las noticias estudiadas. Esto es consecuente con los resultados de diversas investigaciones realizadas en torno al tema del género y el discurso, los cuáles concluyen que en muchos casos los discursos reproducen las relaciones asimétricas de poder existentes entre hombres y mujeres en la sociedad (West, Lazar y Kramae, 2000); en este caso en particular, reproduciendo la visión de los hombres como actores centrales del escenario político y relegando a segundo plano la participación de las mujeres en este.

$\mathrm{Al}$ observar las menciones a Laura Chinchilla en el contenido de las noticias publicadas por los medios seleccionados, se encuentra que también se pueden clasificar siguiendo la misma lógica utilizada para clasificar los titulares. Así, Chinchilla Miranda se presenta de forma activa cuando en el contenido de las noticias se mencionan intervenciones directas de ella, en otras palabras, cuando se le informa a la opinión pública de las acciones que realiza Chinchilla Miranda como respuesta a las diversas problemáticas que surgen en relación con los conflictos sociales. En cuanto a las menciones que pueden clasificarse como pasivas, estas corresponden a diferentes solicitudes o peticiones que distintos actores realizan hacia la presidenta. Estas menciones de tipo pasivas, a su vez, pueden clasificarse en tres tipos: solicitud de posición, las cuales consiste en peticiones de distintos actores sociales a Chinchilla Miranda para que aclare su posición con respecto a los distintos conflictos sociales; solicitudes de diálogo, en las que distintos actores le solicitan a la Presidenta que dialogue con ellos y, por último; solicitudes de intervención, en las que los actores le piden a Laura Chinchilla que tome acciones para solucionar la problemática surgida en torno a los conflictos sociales.

El Cuadro 4 presenta la cantidad de menciones, de Laura Chinchilla, que se realizan en los medios estudiados, según sean una intervención directa (imagen activa) o una solicitud realizada hacia ella (imagen pasiva). En primera instancia se debe mencionar que son mucho más la cantidad de menciones en las que se presenta a Chinchilla Miranda como sujeto pasivo, 88 menciones; mientras que solo 20 menciones la presentan como un sujeto activo. 


\section{CUADRO 4}

Cantidad de menciones de Laura Chinchilla en las noticias

analizadas, según representan una imagen pasiva o activa

\begin{tabular}{|c|c|c|c|c|c|}
\hline & & Diario Extra & La Nación & El País & $\begin{array}{l}\text { Semanario } \\
\text { Universidad }\end{array}$ \\
\hline \multirow{3}{*}{$\begin{array}{l}\text { Imagen } \\
\text { Pasiva }\end{array}$} & Posición & 0 & 1 & 0 & 0 \\
\hline & Diálogo & 2 & 5 & 6 & 2 \\
\hline & Intervención & 18 & 13 & 39 & 2 \\
\hline \multicolumn{2}{|c|}{ Imagen Activa } & 7 & 1 & 7 & 5 \\
\hline
\end{tabular}

Fuente: Elaboración propia, a partir de las noticias encontradas en La Nación, Diario

Extra, El País y el Semanario Universidad, del 8 de mayo del 2010 al 7 de mayo del 2011.

En primera instancia, las menciones que presentan a la presidenta como sujeto pasivo se concentran en solicitudes de intervención. Esto puede ayudar a presentar en el discurso de los medios a Chinchilla Miranda como una figura que no toma acciones o, por lo menos, que no es lo suficientemente activa sobre los conflictos sociales, por lo que otros actores deben -casi de una forma constante- solicitarle directamente que intervenga.

Las siguientes menciones que la presentan como pasiva son solicitudes de diálogo, en estos casos los medios informan que otros actores le solicitan a ella que acuda a conversar y a negociar con ellos en procura de encontrar una solución a dicho conflicto social. Esto puede presentar a Chinchilla Miranda como una figura que no se encuentra relacionándose de forma directa con los actores involucrados en el conflicto social, es decir, busca posicionar ante la opinión pública la idea de que ella es una figura cerrada al diálogo o, al menos, que no busca entrar a dialogar por su propia iniciativa.

\section{CUADRO 5}

Cantidad de menciones de la posición de Laura Chinchilla

sobre los distintos conflictos sociales en las noticias analizadas.

\begin{tabular}{|c|c|c|c|c|}
\hline Posición de Laura Chinchilla & Diario Extra & La Nación & El País & $\begin{array}{l}\text { Semanario } \\
\text { Universidad }\end{array}$ \\
\hline Crucitas & 1 & 4 & 10 & 5 \\
\hline JAPDEVA & 3 & 4 & 4 & 1 \\
\hline $\begin{array}{l}\text { Unión civil de personas del } \\
\text { mismo sexo }\end{array}$ & 0 & 1 & 5 & 1 \\
\hline
\end{tabular}

Fuente: Elaboración propia, a partir de las noticias encontradas en La Nación, Diario Extra, El Pais y el Semanario Universidad, del 8 de mayo del 2010 al 7 de mayo del 2011.

En el caso de solicitudes de posición de Laura Chinchilla, es decir, que ella exprese qué piensa u opina sobre los conflictos sociales, solo ocurre una publicación al respecto. Esto puede deberse a que en las noticias publicadas por los medios sí se le da cierto espacio a informar cuál es la posición de la presidenta (ver Cuadro 5). En este caso es llamativo que ninguno de los medios estudiados menciona en sus noticias cuál es la posición de Chinchilla Miranda respecto al FEES. La razón de lo anterior se debe a que en el contenido de las noticias sobre el FEES se proyecta la imagen de que Chinchilla Miranda busca no intervenir en el asunto y deja esto en manos de sus ministros (Leonardo Garnier y Fernando Herrero), por lo que las menciones sobre ella provienen principalmente de parte de los rectores y las rectoras de las universidades públicas quienes la buscan para que intervenga de forma directa al sentir que no estaba dando resultado la negociación de los ministros (Díaz, 2012b, pp. 46-48). 


\section{CONSIDERACIONES FINALES}

Tras realizar el análisis de contenido de los medios de comunicación seleccionados con respecto a Laura Chinchilla Miranda, se puede afirmar que las personas lectoras de estas noticias pueden preguntarse ¿Dónde está la presidenta? Esto debido a que, como se ha mostrado, la aparición de Chinchilla Miranda en el contenido informativo de los medios es relativamente marginal. Asimismo, cuando se le menciona, se le destaca principalmente como una figura pasiva; en otras palabras, no se visualiza a una presidenta activa, que toma decisiones y ejecuta acciones relacionadas con los problemas y conflictos sociales sobre los que tratan las noticias, sino más bien se le da énfasis a las solicitudes y demandas que otros actores realizan a ella, lo cual deja la impresión de que Laura Chinchilla no busca intervenir o participar en estos asuntos, por lo que los actores sociales involucrados deben buscar diferentes mecanismos para que ella se aproxime a ellos.

No se cuenta con otras investigaciones que hayan analizado o reconstruido el discurso de los medios sobre la imagen de otros presidentes de Costa Rica, por lo cual no es posible comparar si es la coyuntura, la posición política del gobierno o las características de la persona que asume la Presidencia de la República, incluido su género; por lo que se dificulta corroborar si el discurso mediático construido en torno a la figura de Laura Chinchilla Miranda es similar al que se ha construido sobre otros presidentes de Costa Rica, o bien, es producto de la valoración diferenciada de la participación política de las mujeres en la vida política, generada por las estructuras patriarcales presentes en la sociedad (Norris, 1997, p. 77). Sin embargo, otras investigaciones que analizan el caso de las presidentas Cristina Fernández (Argentina) y Dilma Rousseff(Brasil), muestran que el discurso mediático sobre estas se elabora a partir de parámetros patriarcales, los cuales van desde priorizar aspectos de la vida privada, entender la posición política en términos de dependencia masculina o sustantivar la posición política en términos metafórico-reproductivos, entre otros (Ríos, 2017).

Siguiendo con lo anterior, y cómo se mostró en el artículo, es común en los medios analizados representar las opiniones de Chinchilla Miranda a través de terceras personas, generalmente un ministro (hombre). Adicionalmente, en los casos analizados fue común que los medios otorgaran mayor participación en su narrativa a hombres vinculados con el gobierno, que a las mujeres que también intervenían en estos; incluida la propia presidenta. Por lo tanto, es posible inferir que el discurso mediático construido en torno a la imagen de Laura Chinchilla Miranda es producto y, al mismo tiempo, reproduce las estructuras patriarcales imperantes en la sociedad costarricense, especialmente, la idea de que las mujeres son dependientes de los hombres en su actuación; a pesar que estas ocupen roles importantes para la toma de decisiones políticas, incluyendo, la presidencia de la República de Costa Rica.

$\mathrm{Si}$ bien hay que tener mucho cuidado a la hora de generalizar los distintos discursos expresados por $L a$ Nación, Diario Extra, El Pais y el Semanario Universidad, dado que cada uno de estos medios cuenta con su propia agenda mediática y posición política sobre la cual construyen la imagen de la realidad que expresan hacia la opinión pública; el presente texto ha tenido la finalidad de identificar los puntos comunes en el tratamiento de ellos sobre la información relacionada con la Presidenta Chinchilla Miranda. Por lo tanto, si bien las motivaciones e intereses que cada medio posee para presentar la imagen de Laura Chinchilla de una forma determinada pueden variar radicalmente entre ellos, lo que tienen en común es que han colaborado en la construcción de la imagen de una presidenta ausente, por lo menos durante su primer año de gobierno.

Esta imagen de presidenta ausente puede llevar a la población a pensar que Chinchilla Miranda no contaba con las capacidades para ejercer la presidencia, motivo por el cual debe recurrir a intermediarios que se hagan cargo de los conflictos presentes durante su administración. Lo que puede estar detrás de esto es el reflejo de una forma diferente de mandato (o liderazgo) a la que usualmente se encuentra acostumbrada la población costarricense, en la cual la persona que ocupa la presidencia asume un rol central y preponderante en los asuntos públicos, interviniendo directamente -si bien no en todos- en la mayoría de los problemas y conflictos surgidos durante su administración, sin importar si tiene o no la capacidad para lidiar con estos. 
En relación con lo anterior, distintas autoras han indicado que existe la percepción de que las mujeres en puestos políticos ejercen un estilo de liderazgo diferente al realizado por los hombres (Norris, 1997, p. 79 y Uriarte, 1997, pp. 70-14). Así, existe la idea entre amplios sectores de la población de que las mujeres realizarían una política "más sensible y amable", caracterizada por una preeminencia de la cooperación sobre el conflicto, un trato más colaborativo, así como un comportamiento más honesto, ético y compasivo; no obstante, esta percepción parece estar construida más sobre estereotipos de género que por la experiencia empírica obtenida tras el estudio del liderazgo y modos de actuar de diferentes mujeres políticas (Norris, 1997, pp. 79-80).

En el caso de Chinchilla Miranda, algunas autoras han apuntado que su elección como presidenta se debió al carácter de "novedad" que tenía su candidatura para el electorado, esto debido a que presentaba un proyecto político conservador que no la diferenciaba sustancialmente de sus predecesores o la hiciera resaltar en el panorama político costarricense (Flores-Estrada, 2010, pp. 96-97 y Fernández y Oliva, 2012, p. 131); en otras palabras, se puede plantear la tesis de que Chinchilla Miranda fue electa en gran medida debido a los estereotipos que podrían estar presentes en buena parte de la población costarricense con respecto al liderazgo y a las actitudes que tienen las mujeres en el espacio político. Lo anterior, puede llevar a sesgar la percepción de la población sobre la actuación de Chinchilla Miranda, ya que miden esta a partir de la expectativa del cumplimiento de determinados comportamientos basados más en estereotipos que en expectativas reales y en lo planteado por la presidenta durante la campaña política.

Tomado esto en consideración, sería posible pensar que tanto los medios de comunicación como los grupos sociales involucrados en los conflictos analizados esperaban en Chinchilla Miranda una actitud más conciliatoria, que sostuviera una posición más abierta al diálogo y fuera una figura que interviniera de forma más activa en las situaciones conflictivas. Por lo tanto, la imagen proyectada por la prensa escrita con relación a Laura Chinchilla puede deberse al hecho de que ella no cumpliera con estas expectativas o, al menos, no lo hizo de la forma esperada por estos. De esta forma, se estaría partiendo de la condición de Chinchilla como mujer para construir y valorar sus acciones en el discurso de los medios y no, propiamente, por las acciones mismas y sus resultados.

Por lo tanto, durante el primer año de la administración Chinchilla Miranda los medios de comunicación analizados proyectan a la opinión pública la imagen de una presidenta pasiva, que no actúa de forma inmediata ante los conflictos sociales, sino que debe ser convocada por otros actores para pronunciarse al respecto y que se mantiene en la medida de lo posible al margen de los conflictos. Tras considerar esto podríamos preguntarnos qué tanto la percepción de "ingobernabilidad" y de mala gestión de Chinchilla, presente en sectores importantes de la población, se debe a las acciones, omisiones y errores cometida por ella, y que tanto se debe a lo que parece ser una construcción sistemática de los medios de comunicación de una imagen de Chinchilla la cual, si bien no puede calificarse abiertamente como negativa, puede llevar a la opinión pública a considerarla como poco competente para el ejercicio de la presidencia de la República de Costa Rica.

\section{Agradecimientos}

Agradezco a las personas evaluadoras anónimas sus valiosos comentarios y observaciones, las cuales sirvieron para mejorar el presente artículo. Sin embargo, cualquier error u omisión que pueda existir en el presente texto es de mi exclusiva responsabilidad como autor.

\section{REFERENCIAS}

Abarca, A. (2012). El Análisis de Contenido. En A. Abarca, C. Rojas, G. Sibaja, F. Alpízar. (Eds.), Técnicas cualitativas de investigación (pp.193-248). San José: Editorial UCR. 
Dr. José Andrés Díaz González. ¿Dónde está la presidenta? la construcción de la imagen de Laura Ch...

Díaz, J. A. (2012a). Prensa escrita ante el referéndum acerca de la Unión de personas del mismo sexo (2010). Revista Rupturas, 2(2), 294-326. doi: https://doi.org/10.22458/rr.v2i2.182

Díaz, J. A. (2012b). El discurso de la prensa escrita sobre la negociación del FEES (2010). Revista Universidad en Diálogo, No Especial, 115-146. Recuperado de https://www.revistas.una.ac.cr/index.php/dialogo/article/view/ 6349

Fernández, F. (2005). Valores del léxico en el discurso informativo: Enfoque crítico. Estudios de Sociolingüistica, 6(2), 219-241.

Fernández, M. y Oliva, D. (2012). Presidentas latinoamericanas e igualdad de género: un camino sinuoso. Nueva Sociedad, 240, 119-133. Recuperado de https://bit.ly/2R9B23y

Flores-Estrada, M. (2010). La campaña de Laura Chinchilla y las mujeres ¿Oportunismo o compromiso con un nuevo pacto sexual? Revista de Ciencias Sociales, 130(4), 85-99.

Krippendorff, K. (1997). Metodología de análisis de contenido. Teoria y Práctica. Barcelona: Paidós

Leñero, V. y Marín, C. (1986). Manual de Periodismo. México DF: Editorial Grijalbo.

Morales, J. (2007). Argentina, el sistema de los Kirchner: Adiós a los viejos partidos. Política Exterior, 120(21), 31-34. Recuperado de https://bit.ly/2Y63VyU

Navia, P. (2007). ¿Qué le pasó a Bachelet? Nueva Sociedad, 214, 4-14. Recuperado de https://nuso.org/articulo/que -le-paso-a-bachelet/

Norris, P. (1997). Las Mujeres Políticas: ¿Un nuevo estilo de liderazgo? En A. Elizondo y E. Uriarte (Coords.), Mujeres en Política. Análisis y práctica (pp. 76-96). Barcelona: Editorial Ariel, S.A.

Ríos, J. (2017). Liderazgo político y patriarcado mediático: las imágenes políticas de Cristina Fernández y Dilma Rousseff. Revista de Investigaciones Politicas y Sociológicas, 16(1), 65-85. Recuperado de https://www.redalyc.o $\mathrm{rg} /$ articulo.oa?id=38051452003

Uriarte, E. (1997). Las mujeres en las élites políticas. En A. Elizondo y E. Uriarte (Coords.), Mujeres en Política. Análisis $y$ práctica (pp. 182-200). Barcelona: Editorial Ariel, S.A.

Van Dijk, T. (1999). Ideologia. Barcelona: Editorial Gedisa.

Van Dijk, T. (2000). El discurso como interacción en la sociedad. En T. Van Dijk (Comp.), El discurso como interacción social. Estudios sobre el discurso: Una introducción multidisciplinaria. (pp. 19-66). Barcelona: Editorial Gedisa.

Van Dijk, T. (2003a). La multidisciplinaridad del análisis crítico del discurso: un alegato en favor de la diversidad. En M. Meyer y R. Wodak (Comps.), Métodos de análisis crítico del discurso. (pp. 143-177). Barcelona: Editorial Gedisa.

Van Dijk, T. (2003b). Élite, discurso y racismo. Barcelona: Editorial Gedisa.

West, C., Lazar, M. M. y Kramaer, C. (2000). El género en el discurso. En T. van Dijk (Comp.), El discurso como interacción social. Estudios sobre el discurso: Una introducción multidisciplinaria. (pp. 179-212). Barcelona: Editorial Gedisa.

\section{Notas}

1. Para la codificación de la información se procedió a utilizar dos programas informáticos, el primero, Atlas.ti, el cual permite identificar los segmentos de texto relacionados con Chinchilla Miranda, al mismo tiempo que se pueden etiquetar a partir de los temas principales que contienen, para su posterior conteo. El segundo, Wordsmith, es un software que permite el conteo de las palabras presentes en los textos, con el propósito de identificador cuales palabras son más (o menos) utilizadas en su contenido.

Todos los derechos reservados. Universidad de Costa Rica. Esta revista se encuentra licenciada con Creative Commons. Reconocimiento-NoComercial-SinObraDerivada 3.0 Costa Rica. Correo electrónico: humanidades@ucr.ac.cr/Sitioweb: http: //revistas.ucr.ac.cr/index.php/humanidades CC BY-NC-ND 